\title{
Reproducibility of Lobar Perfusion and Ventilation Quantification Using SPECT/CT Segmentation Software in Lung Cancer Patients
}

\author{
Karine Provost ${ }^{1}$, Antoine Leblond ${ }^{1}$, Annie Gauthier-Lemire ${ }^{1}$, Édith Filion ${ }^{2}$, Houda Bahig ${ }^{2}$, and Martin Lord ${ }^{1}$ \\ ${ }^{I}$ Department of Nuclear Medicine, Centre Hospitalier de l'Université de Montréal, Montréal, Quebec, Canada; and ${ }^{2}$ Department of \\ Radiation Oncology, Centre Hospitalier de l'Université de Montréal, Montréal, Quebec, Canada
}

Planar perfusion scintigraphy with 99mTc-labeled macroaggregated albumin is often used for pretherapy quantification of regional lung perfusion in lung cancer patients, particularly those with poor respiratory function. However, subdividing lung parenchyma into rectangular regions of interest, as done on planar images, is a poor reflection of true lobar anatomy. New tridimensional methods using SPECT and SPECT/CT have been introduced, including semiautomatic lung segmentation software. The present study evaluated inter- and intraobserver agreement on quantification using SPECT/CT software and compared the results for regional lung contribution obtained with SPECT/CT and planar scintigraphy. Methods: Thirty lung cancer patients underwent ventilation-perfusion scintigraphy with $99 \mathrm{~m}$ Tc-macroaggregated albumin and 99mTc-Technegas. The regional lung contribution to perfusion and ventilation was measured on both planar scintigraphy and SPECT/CT using semiautomatic lung segmentation software by 2 observers. Interobserver and intraobserver agreement for the SPECT/CT software was assessed using the intraclass correlation coefficient, BlandAltman plots, and absolute differences in measurements. Measurements from planar and tridimensional methods were compared using the paired-sample $t$ test and mean absolute differences. Results: Intraclass correlation coefficients were in the excellent range (above 0.9) for both interobserver and intraobserver agreement using the SPECT/CT software. Bland-Altman analyses showed very narrow limits of agreement. Absolute differences were below $2.0 \%$ in $96 \%$ of both interobserver and intraobserver measurements. There was a statistically significant difference between planar and SPECT/CT methods $(P<0.001)$ for quantification of perfusion and ventilation for all right lung lobes, with a maximal mean absolute difference of $20.7 \%$ for the right middle lobe. There was no statistically significant difference in quantification of perfusion and ventilation for the left lung lobes using either method; however, absolute differences reached $12.0 \%$. The total right and left lung contributions were similar for the two methods, with a mean difference of $1.2 \%$ for perfusion and $2.0 \%$ for ventilation. Conclusion: Quantification of regional lung perfusion and ventilation using SPECT/CT-based lung segmentation software is highly reproducible. This tridimensional method yields statistically significant differences in measurements

\footnotetext{
Received Jan. 31, 2017; revision accepted Apr. 11, 2017.

For correspondence or reprints contact: Karine Provost, Centre Hospitalier de I'Université de Montréal, 3840 rue Saint-Urbain, Montréal, QC, Canada H2W1T8.

E-mail: provost.karine@gmail.com

Published online Apr. 13, 2017.

COPYRIGHT (c) 2017 by the Society of Nuclear Medicine and Molecular Imaging.
}

for right lung lobes when compared with planar scintigraphy. We recommend that SPECT/CT-based quantification be used for all lung cancer patients undergoing pretherapy evaluation of regional lung function.

Key Words: lobar quantification; pulmonary perfusion; pulmonary ventilation; scintigraphy; SPECT/CT

J Nucl Med Technol 2017; 45:185-192

DOI: 10.2967/jnmt.117.191056

$\mathbf{Q}$ uantification of regional lung perfusion with ${ }^{99 \mathrm{~m}} \mathrm{Tc}-$ labeled macroaggregated albumin is commonly used to evaluate pulmonary function in patients with lung cancer before volume reduction surgery $(1,2)$ or radiotherapy $(3)$. Quantification allows estimation of the impact of treatment on forced expiratory volume in $1 \mathrm{~s}$ in those with borderline pretherapy lung function $(4,5)$. The current most widely used method for quantification of regional lung function uses planar perfusion scintigraphy. In that method, the lung parenchyma is subdivided into 6 equal-sized rectangular regions of interest (ROIs). The relative percentage of counts in each lung region is calculated from the geometric mean on anterior and posterior views, thus approximating the lobar contribution. However, rectangular ROIs are a poor representation of true lobar anatomy (5).

With the advent of hybrid imaging, new methods of tridimensional lung segmentation using SPECT and SPECT/CT have been introduced, offering anatomically based quantification of the lobar contribution for perfusion studies (6-8) or combined ventilation-perfusion studies $(9,10)$. Recently, a semiautomatic lung segmentation software program has been developed for SPECT/CT (Hermes Hybrid 3D Lung Lobe Quantification; Hermes Medical Solutions). However, the reproducibility of measurements obtained with this software in a clinical setting has not been studied, nor has the feasibility of SPECT/CT quantification in a group of patients with a high prevalence of underlying lung disease, such as nonsurgical candidates undergoing radiotherapy.

In the present study, we evaluated the feasibility and the inter- and intraobserver agreement of lung quantification using this software, and we compared the regional lung 
TABLE 1

Patient Characteristics

\begin{tabular}{|c|c|c|c|c|c|}
\hline Patient $(n=30)$ & Age (y) & Sex & Primary tumor site & Underlying lung disease & Central deposition of Technegas \\
\hline 1 & 79 & $M$ & LUL & Severe emphysema & Present \\
\hline 2 & 80 & $\mathrm{~F}$ & LUL & None & Absent \\
\hline 3 & 61 & M & LLL & None & Absent \\
\hline 4 & 79 & $\mathrm{~F}$ & LUL & None & Present \\
\hline 5 & 62 & $\mathrm{~F}$ & RLL & Moderate emphysema & Absent \\
\hline 6 & 53 & $\mathrm{~F}$ & RML & Severe emphysema & Present \\
\hline 7 & 73 & $M$ & LUL & Severe emphysema & Present \\
\hline 8 & 70 & M & RLL & Severe emphysema & Present \\
\hline 9 & 74 & $\mathrm{M}$ & RUL & Severe emphysema & Absent \\
\hline 10 & 79 & $\mathrm{~F}$ & LUL & None & Absent \\
\hline 11 & 52 & $\mathrm{~F}$ & Mediastinum & None & Absent \\
\hline 12 & 65 & $M$ & LUL & Severe emphysema & Absent \\
\hline 13 & 63 & M & RLL & Moderate emphysema & Absent \\
\hline 14 & 75 & $\mathrm{~F}$ & RUL & None & Absent \\
\hline 15 & 60 & $\mathrm{~F}$ & RUL & None & Absent \\
\hline 16 & 70 & M & RLL & Severe emphysema & Absent \\
\hline 17 & 69 & M & LLL & Severe emphysema & Present \\
\hline 18 & 69 & M & LUL & Moderate emphysema & Absent \\
\hline 19 & 56 & M & RUL & Severe emphysema & Present \\
\hline 20 & 75 & M & LUL & Multifocal infiltrates & Absent \\
\hline 21 & 69 & M & LUL & Severe emphysema & Present \\
\hline 22 & 69 & $\mathrm{~F}$ & RLL & None & Absent \\
\hline 23 & 65 & $\mathrm{~F}$ & RUL and RLL & None & Absent \\
\hline 24 & 80 & $M$ & LUL & Moderate emphysema & Absent \\
\hline 25 & 74 & $M$ & RLL & Moderate emphysema & Absent \\
\hline 26 & 70 & $\mathrm{M}$ & LLL & Severe emphysema & Present \\
\hline 27 & 64 & $\mathrm{~F}$ & RLL & Moderate emphysema & Present \\
\hline 28 & 56 & $\mathrm{~F}$ & RLL & None & Absent \\
\hline $29^{*}$ & 55 & $\mathrm{~F}$ & LUL & Severe emphysema & Present \\
\hline 30 & 67 & $\mathrm{~F}$ & LUL & None & Present \\
\hline
\end{tabular}

${ }^{\star}$ Excluded patient.

$\mathrm{LUL}=$ left upper lobe; $\mathrm{LLL}=$ left lower lobe; $\mathrm{RLL}=$ right lower lobe; $\mathrm{RML}=$ right middle lobe; $\mathrm{RUL}=$ right upper lobe.

contribution results with those of traditional planar methods for ventilation-perfusion studies.

\section{MATERIALS AND METHODS}

We included 30 consecutive lung cancer patients (14 women and 16 men with a mean age $68.2 \mathrm{y}$; range, 52-80 y) referred to our Nuclear Medicine Department for preradiotherapy evaluation of lung function from April 2015 to June 2016. The institutional review board approved this study, and all subjects signed an informed consent form. The patient characteristics are detailed in Table 1.

Ventilation and perfusion scintigraphy were performed on the same day with inhalation of ${ }^{99 \mathrm{~m} T c-T e c h n e g a s ~(C y c l o m e d i c a ; ~}$ mean dose, $577 \mathrm{MBq}$ placed in a crucible) and intravenous injection of ${ }^{99 \mathrm{~m}} \mathrm{Tc}$-macroaggregated albumin (mean dose, 292 $\mathrm{MBq}$ ). The ratio of perfusion counting rate to ventilation counting rate was higher than 4 in all patients. All studies were performed on a dedicated SPECT/CT camera (Discovery NM/CT 670; GE Healthcare). A SPECT-only acquisition was performed for the ventilation study, whereas a SPECT/CT acquisition with attenuation correction was performed for the perfusion study. Planar anterior and posterior acquisitions were obtained for both the ventilation and the perfusion studies. The SPECT acquisition parameters included a $20 \%$ energy window centered at $140 \mathrm{keV}$, a
$128 \times 128$ matrix, a total acquisition of 60 frames over $360^{\circ}$, with $28 \mathrm{~s}$ per projection for the ventilation acquisition and $17 \mathrm{~s}$ for the perfusion acquisition. Acquisitions were performed using a lowenergy, high-resolution parallel-hole collimator and a zoom factor of 1.28. SPECT reconstructions were performed using iterative tridimensional ordered-subsets expectation maximization (HRECON, version 1.1C; Hermes Medical Solutions) with 3 iterations, 6 subsets, and a tridimensional gaussian filter $(1.25 \mathrm{~cm}$ in full width at half maximum). A helical CT acquisition was obtained during free breathing immediately before the perfusion SPECT acquisition. The CT parameters included a voltage of $120 \mathrm{kV}$, a current of $150 \mathrm{~mA}$, a 0.5 -s rotation time, a pitch of $1.375,16 \times$ $0.625 \mathrm{~mm}$ collimation, and reconstruction at a $1.25-\mathrm{mm}$ slice thickness using filtered backprojection and a soft-tissue filter. There was no intravenous contrast injection. The estimated effective doses were $3 \mathrm{mSv}$ for SPECT acquisitions and $2 \mathrm{mSv}$ for a single CT acquisition. SPECT and SPECT/CT images were reviewed for adequate coregistration. Planar ventilation images were visually assessed for central deposition of Technegas.

For planar scintigraphy, quantification was performed using Hermes Hybrid Viewer lung quantification software (Hermes Medical Solutions), which subdivides each lung into 3 rectangular ROIs and computes the relative contribution (percentage) of each ROI from the geometric mean of counts on anterior and posterior 


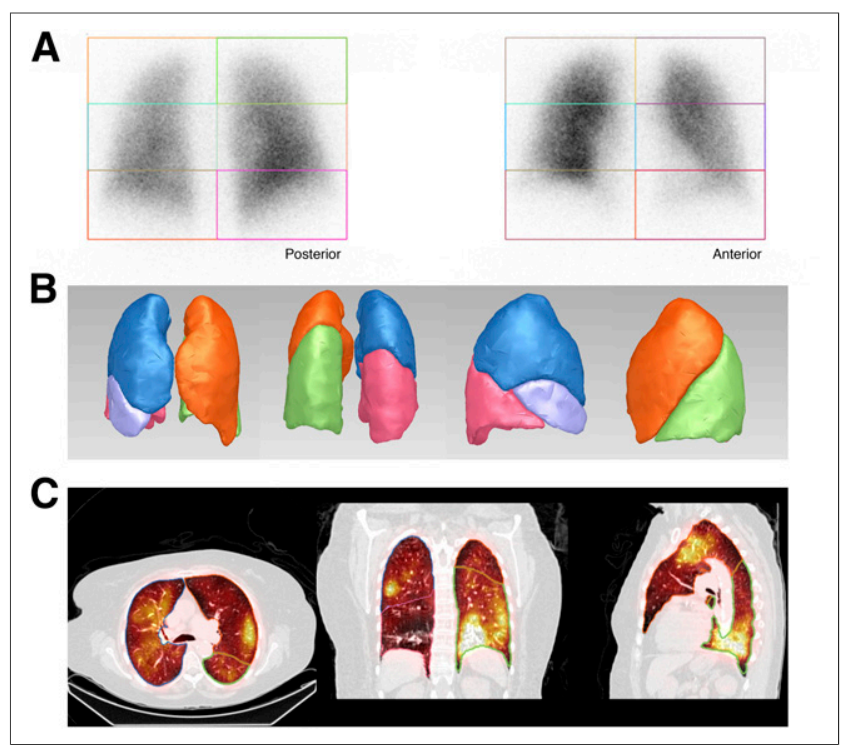

FIGURE 1. (A) Quantification of perfusion using planar method. (B and C) Quantification of perfusion with semiautomatic SPECT/ CT-based lung segmentation software (CT-based volumes of interest $[\mathrm{C}]$ with tridimensional rendering $[\mathrm{B}])$.

views (Fig. 1A). For SPECT and SPECT/CT, tridimensional quantification was performed using Hermes Lung Lobe Quantification with Hybrid 3D. The workflow in this software consists of, first, automatic CT-based lung volume detection through a region-growing algorithm; second, semiautomatic delimitation of lung fissures (the user defines 6 points along each lung fissure on multiple reconstructed CT slices in the sagittal plane); third, software computation of 5 volumes of interest, one for each lobe (right upper, right middle, right lower, left upper, and left lower).

Ventilation and perfusion studies are coregistered to CT with a mutual-information algorithm and an identical frame of reference, respectively. Volumes of interest are applied to coregistered ventilationperfusion SPECT data, and the relative contribution (percentage) of each lobe is computed for both ventilation and perfusion. A tridimensional rendering of CT-based volumes of interest is displayed along with the quantification results (Figs. 1B and 1C).

SPECT/CT-based segmentation was performed by observer 1 for both ventilation and perfusion studies for all patients and by observer 2 once for perfusion. Observer 1 repeated the measurements at least $1 \mathrm{wk}$ later to minimize recall bias. The observers were masked to any previous measurement.

Interobserver and intraobserver agreement on the tridimensional quantification of perfusion and ventilation, respectively, was assessed using 2-way random, absolute-agreement, singlemeasures intraclass correlation coefficients (11).

For both the interobserver and the intraobserver analyses, absolute differences between measurements were calculated, as well as the mean absolute difference for each lobe. Bland-Altman plots were also done for interobserver analysis (12).

The quantification results for the left middle third on planar scintigraphy were redistributed equally between the left upper and left lower thirds to allow comparison with the left upper and left lower lobes, respectively, for the tridimensional method (Fig. 2). The quantification results for the right lung ROIs on planar scintigraphy were compared directly (i.e., upper third with right upper lobe, middle third with right middle lobe, and lower third with right

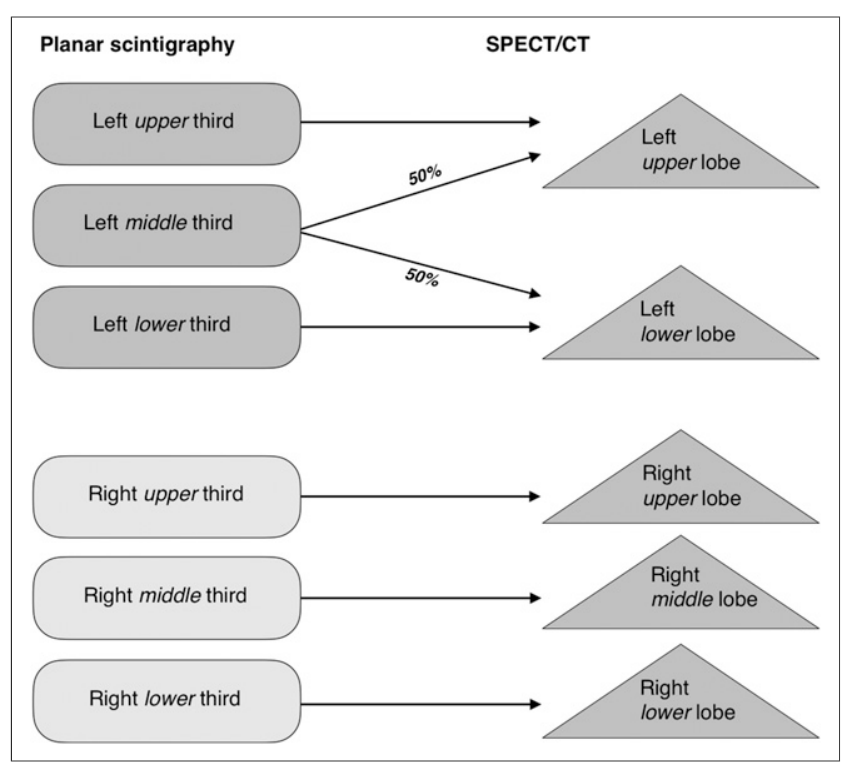

FIGURE 2. Schematic representation for comparison of quantification measurements on planar scintigraphy and SPECT/CT.

lower lobe). Comparison of results for total right and total left lungs, as well as lobar quantification obtained by planar and SPECT/CT studies (using results from observer 1) was performed using pairedsample $t$ tests for both ventilation and perfusion. Subgroup analysis was performed for patients with and without central deposition of Technegas on the planar ventilation study. $P$ values of less than 0.05 were considered statistically significant. Mean absolute differences for the results of both methods were also calculated. Statistical analyses were performed with SPSS (version 24.0.0.0; IBM).

\section{RESULTS}

Semiautomatic segmentation using the SPECT/CT-based software was feasible in 29 of the 30 patients, taking on average 5-10 min per patient. We excluded one patient because automatic detection of the lung contour failed.

Mean measurements obtained by planar imaging and SPECT/CT, mean absolute differences between the two methods, and $P$ values from paired-sample $t$ tests comparing

TABLE 2

Comparison of Quantification Values on Perfusion Studies

\begin{tabular}{|c|c|c|c|c|}
\hline Site & Planar & SPECT/CT & $\begin{array}{l}\text { Absolute } \\
\text { difference }\end{array}$ & $P$ \\
\hline Total right lung & 54.8 & 55.1 & $1.2(0-3.4)$ & 0.15 \\
\hline Total left lung & 45.2 & 44.9 & $1.2(0-3.4)$ & 0.15 \\
\hline $\begin{array}{l}\text { Right upper } \\
\text { lobe }\end{array}$ & 11.9 & 22.2 & $10.7(2.1-22.8)$ & $<0.001$ \\
\hline $\begin{array}{l}\text { Right middle } \\
\text { lobe }\end{array}$ & 29.4 & 8.7 & $20.7(11.1-36.0)$ & $<0.001$ \\
\hline Right lower lobe & 13.5 & 24.6 & $11.1(0.3-23.6)$ & $<0.001$ \\
\hline Left upper lobe & 22.7 & 23.9 & $3.2(0-10.6)$ & 0.63 \\
\hline Left lower lobe & 21.5 & 21.3 & $3.7(0-10.8)$ & 0.31 \\
\hline
\end{tabular}

Data are mean, with range in parentheses. 


\begin{tabular}{|c|c|c|c|c|c|c|c|c|}
\hline \multirow[b]{2}{*}{ Site } & \multirow[b]{2}{*}{ Planar } & \multirow[b]{2}{*}{ SPECT/CT } & \multicolumn{2}{|c|}{ All patients $(n=29)$} & \multicolumn{2}{|c|}{$\begin{array}{l}\text { No central deposition } \\
\qquad(n=18)\end{array}$} & \multicolumn{2}{|c|}{ Central deposition $(n=11)$} \\
\hline & & & Absolute difference & $P$ & Absolute difference & $P$ & Absolute difference & $P$ \\
\hline Total right lung & 54.6 & 56.0 & $2.0(0-8.8)$ & 0.01 & $1.9(0.1-8.8)$ & 0.13 & $2.2(0.4-6.0)$ & 0.02 \\
\hline Total left lung & 45.4 & 44.0 & $2.0(0-8.8)$ & 0.01 & $1.9(0.1-8.8)$ & 0.13 & $2.2(0.4-6.0)$ & 0.02 \\
\hline Right upper lobe & 11.8 & 20.6 & $8.8(0.2-19.2)$ & $<0.001$ & $9.1(0.2-19.2)$ & $<0.001$ & $8.3(4.9-13.6)$ & $<0.001$ \\
\hline Right middle lobe & 27.3 & 9.3 & $18.0(10.6-29.7)$ & $<0.001$ & $17.9(12.5-27.0)$ & $<0.001$ & $18.2(10.6-29.7)$ & $<0.001$ \\
\hline Right lower lobe & 15.5 & 26.1 & $10.9(0.2-12.0)$ & $<0.001$ & $10.4(0.2-17.3)$ & $<0.001$ & $11.8(2.4-27.3)$ & $<0.001$ \\
\hline Left upper lobe & 21.7 & 22.3 & $3.6(0-12.0)$ & 0.82 & $3.2(0-12.0)$ & 0.87 & $4.3(0.1-11.5)$ & 0.63 \\
\hline Left lower lobe & 22.4 & 22.0 & $3.5(0-11.1)$ & 0.22 & $3.4(0-11.1)$ & 0.27 & $3.6(0.1-9.8)$ & 0.57 \\
\hline
\end{tabular}

Data are mean, with range in parentheses. Subgroup analyses were performed for patients with and without central deposition of Technegas.

the two methods are shown in Tables 2 and 3 for perfusion and ventilation, respectively.

The right/left lung differentials obtained by the planar and tridimensional methods did not significantly differ for the perfusion studies (Table 2) but did for the ventilation studies (Table 3). However, no statistically significant difference was found when only the subgroup of patients without central deposition of Technegas was considered $(n=18)$ (Table 3).

For lobar quantification of both perfusion and ventilation, there was a statistically significant difference in values obtained by the planar and tridimensional methods for the right upper, right middle, and right lower lobes, whereas there was no statistically significant difference for the left upper and left lower lobes, as shown in Tables 2 and 3. For the left lung lobes, one third of patients had an absolute difference of more than $5.0 \%$ in quantification measurements between the two methods.

When considering only the lobe of clinical interest for each patient (e.g., left upper lobe quantification for a patient with left upper lobe tumor), there was a statistically significant difference in measurements obtained by SPECT/CT and planar methods $(P=0.04)$, with a mean absolute difference of $7.1 \%$ for perfusion (minimum, $0.2 \%$; maximum, $22.8 \%$ ). For both perfusion and ventilation, absolute differences between total right and left lung contribution measured with planar and tridimensional methods were very small. However, absolute differences were much higher for individual lobar values, especially for right lung lobes, with a maximal absolute difference for the right middle lobe of $36.0 \%$ for perfusion and $29.7 \%$ for ventilation (Tables 2 and 3).

Comparison of interobserver measurements revealed very small mean absolute differences $(0.3 \%-1.0 \%)$, as well as intraclass correlation coefficients in the excellent range (Table 4). Bland-Altman plots showed narrow limits of agreement for all measurements (Fig. 3).

Intraclass correlation coefficients for intraobserver measurements were also excellent $(>0.9)$ for all lobes and for total right and left lungs, with very small mean absolute differences between repeated measurements $(0.3 \%-1.3 \%)$ (Table 5).

Absolute differences of less than $2.0 \%$ were found in $96 \%$ of repeated interobserver and intraobserver measurements (Figs. 4 and 5).

\section{DISCUSSION}

Quantification of lobar perfusion and ventilation using SPECT and SPECT/CT with a semiautomatic segmentation algorithm has excellent inter- and intraobserver agreement. This method yields statistically and clinically significant differences when compared with segmentation with planar scintigraphy for the right lung lobes, with an absolute

TABLE 4

Interobserver Measurements for Quantification on SPECT/CT Perfusion Studies

\begin{tabular}{lcccc}
\hline \multicolumn{1}{c}{ Site } & Mean & Mean absolute difference & Intraclass correlation coefficient & SEM for ICC \\
\hline Total right lung & $55.1(35.8-76.9)$ & $0.3(0-1.6)$ & $0.998(0.996-0.999)$ & 0.810 \\
Total left lung & $44.9(23.1-64.2)$ & $0.3(0-1.6)$ & $0.998(0.996-0.999)$ & 0.810 \\
Right upper lobe & $21.9(1.8-38.5)$ & $0.7(0-2.1)$ & $0.993(0.984-0.997)$ & 1.321 \\
Right middle lobe & $8.7(1.7-14.1)$ & $1.0(0-3.2)$ & $0.922(0.841-0.963)$ & 1.832 \\
Right lower lobe & $24.6(13.1-39.3)$ & $0.9(0-3.5)$ & $0.984(0.966-0.992)$ & 1.830 \\
Left upper lobe & $23.8(6.0-36.0)$ & $0.3(0-1.7)$ & $0.990(0.979-0.995)$ & 1.278 \\
Left lower lobe & $21.5(8.1-33.8)$ & $0.6(0-3.3)$ & $0.989(0.977-0.995)$ & 1.390
\end{tabular}

Data in parentheses are range for mean and mean absolute difference and are $95 \%$ confidence interval for ICC. 


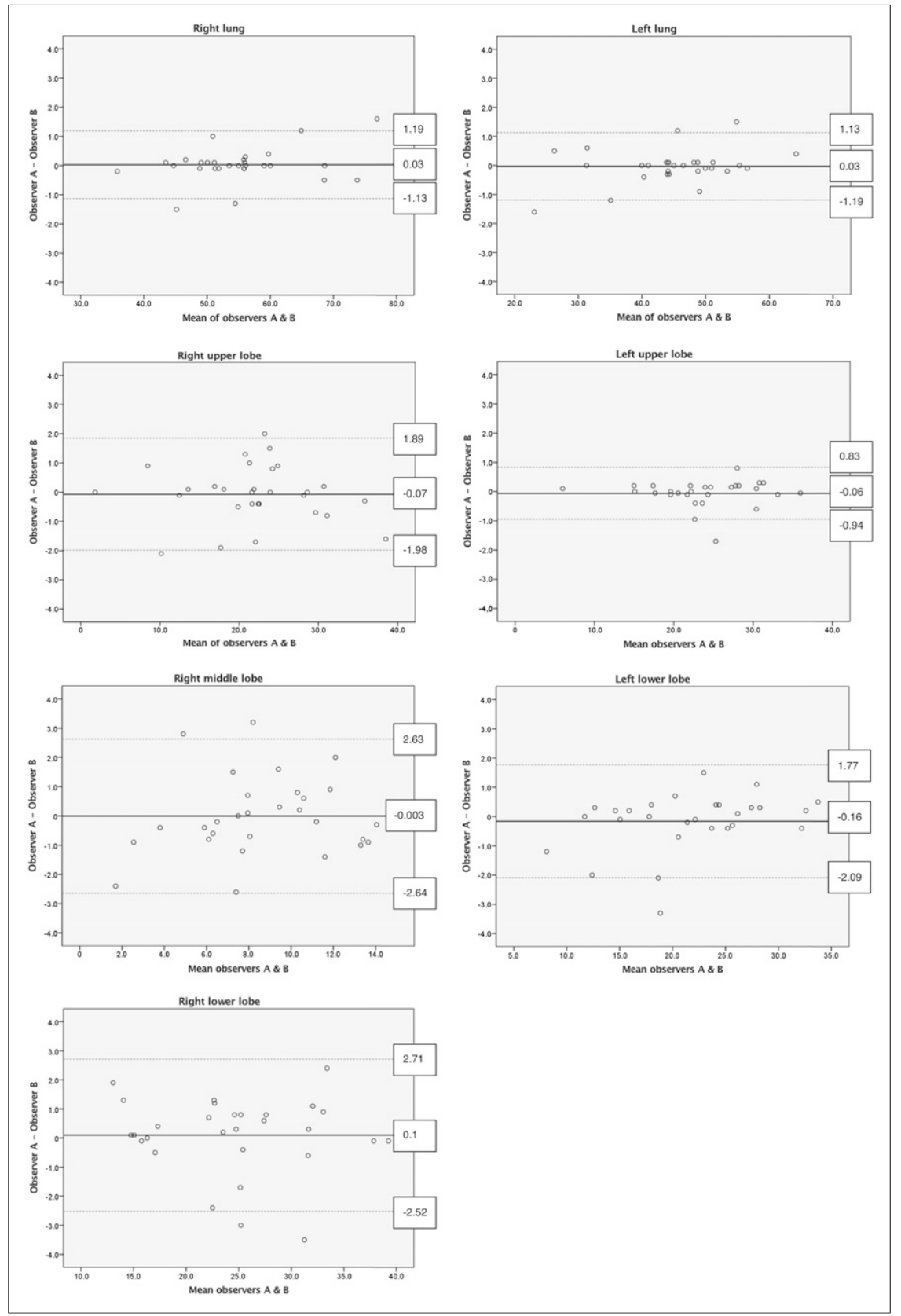

FIGURE 3. Bland-Altman plots for interobserver measurements of total lung and lobar perfusion using SPECT/CT. difference of up to $36.0 \%$. Although differences in values for the left lung lobes were not statistically significant, they can be considered clinically significant, with absolute differences of greater than $5.0 \%$ in one third of patients and maximal absolute differences of $12.0 \%$. We can hypothesize that such differences could alter the clinical management of certain patients.

In practice, the left lung is often subdivided into 3 ROIs when planar images are used for quantification. For the purpose of this study, the contribution of the left middle ROI was redistributed equally to the left upper and left lower thirds. This redistribution more closely approximates true left lung anatomy, with more or less equal-sized upper and lower lobes. This may explain the lack of statistically significant differences when we compare quantification results for the left lung lobes from planar and SPECT/CT segmentation techniques.

Delineation of volumes of interest from the CT acquisition more accurately represents individual patient anatomy. Therefore, ventilation-perfusion quantification obtained from those volumes of interest is more likely to represent the true lobar contribution to perfusion and ventilation than the arbitrarily defined rectangular subdivisions drawn on planar scintigraphy. Furthermore, on planar images, extraneous activity may not be easily excluded from the quantification. Examples include tracheobronchial deposition of ${ }^{99 \mathrm{~m}} \mathrm{Tc}-\mathrm{Technegas}$, thyroid and gastric activity from free pertechnetate, and residual vascular activity on 
TABLE 5

Intraobserver Measurements for Quantification on SPECT/CT Ventilation Studies

\begin{tabular}{lcccc}
\hline \multicolumn{1}{c}{ Site } & Mean & Mean absolute difference & Intraclass correlation coefficient & SEM for ICC \\
\hline Total right lung & $56.0(46.3-74.0)$ & $0.4(0-1.6)$ & $0.997(0.994-0.999)$ & 0.798 \\
Total left lung & $44.0(26.1-53.7)$ & $0.4(0-1.7)$ & $0.997(0.994-0.999)$ & 0.798 \\
Right upper lobe & $20.7(7.7-33.8)$ & $1.3(0-3.5)$ & $0.956(0.910-0.979)$ & 2.312 \\
Right middle lobe & $9.3(0.5-14.6)$ & $0.9(0-3.4)$ & $0.943(0.882-0.973)$ & 1.661 \\
Right lower lobe & $26.0(13.9-42.2)$ & $0.8(0-2.3)$ & $0.989(0.977-0.995)$ & 1.443 \\
Left upper lobe & $22.3(7.3-33.1)$ & $0.3(0-1.3)$ & $0.989(0.977-0.995)$ & 1.174 \\
Left lower lobe & $22.0(10.0-33.3)$ & $0.6(0-3.3)$ & $0.989(0.976-0.995)$ & 1.205
\end{tabular}

Data in parentheses are range for mean and mean absolute difference and are 95\% confidence interval for ICC.

99mTc-macroaggregated albumin studies. Additionally, in some cases, the right and left lungs cannot be easily separated with the preset rectangular ROIs (Fig. 6). Tridimensional segmentation with SPECT/CT overcomes these limitations by considering only the activity corresponding to true lung parenchyma. For these reasons, we believe that planar-based quantification should be abandoned in favor of SPECT/CT or SPECT in centers without access to hybrid cameras.

Several studies have investigated manual tridimensional lung segmentation techniques using SPECT or SPECT/CT for both preoperative $(6-8,10,13-15)$ and preradiotherapy $(16,17)$ patients. Only a few studies investigated semiautomatic algorithms for total lung and lobar segmentation using SPECT/CT in preoperative patients $(9,18)$. No studies had previously compared results to those obtained with planar scintigraphy, or evaluated interobserver and intraobserver agreement on patient studies. In our study, we demonstrated the excellent reproducibility of the semiautomatic segmentation technique in a population with a high prevalence $(63 \%)$ of underlying lung disease, which makes visualization of lung anatomy more challenging. We can hypothesize that we would obtain even more reproducible results in patients with unaltered lung parenchyma, such as most preoperative patients.

Quantification based on perfusion scintigraphy only, as opposed to combined ventilation-perfusion scintigraphy, has been used by some groups $(7,8,18)$, with few studies having investigated the role of ventilation scintigraphy on prediction of posttreatment forced expiratory volume in $1 \mathrm{~s}(9,10)$. However, at our institution, combined ventilation-perfusion scintigraphy is routinely performed to exclude underlying thromboembolic disease in cancer patients referred for quantitative perfusion lung scintigraphy. In our cohort, one patient had an incidental finding of pulmonary emboli.

Multiple non-nuclear medicine imaging techniques have been described for evaluation of lung perfusion, including MRI, CT pulmonary angiography, and doubleenergy CT with parenchymal iodine mapping (19). Although these techniques are accurate, widespread use for perfusion quantification has been restricted by limited accessibility, as well as contraindications and the inherent risk of iodine contrast injection. These CT-based techniques also have substantially higher radiation doses to patients than do nuclear medicine techniques.

The semiautomatic lung segmentation software used in this study allows simple and rapid quantification of both perfusion and ventilation studies in a single streamed workflow, taking on average 5-10 min per patient. Some limitations of this technique include occasional difficulty in visualizing lung fissures on free-breathing CT, although this occurred in a minority of patients and did not significantly influence inter- or intraobserver agreement, as shown in our results. In our study, CT was acquired during free breathing to match perfusion acquisition. In patients with suboptimal fissure visualization,

FIGURE 4. Absolute differences in interobserver measurements of total lung (A) and lobar (B) contribution using SPECT/CT.
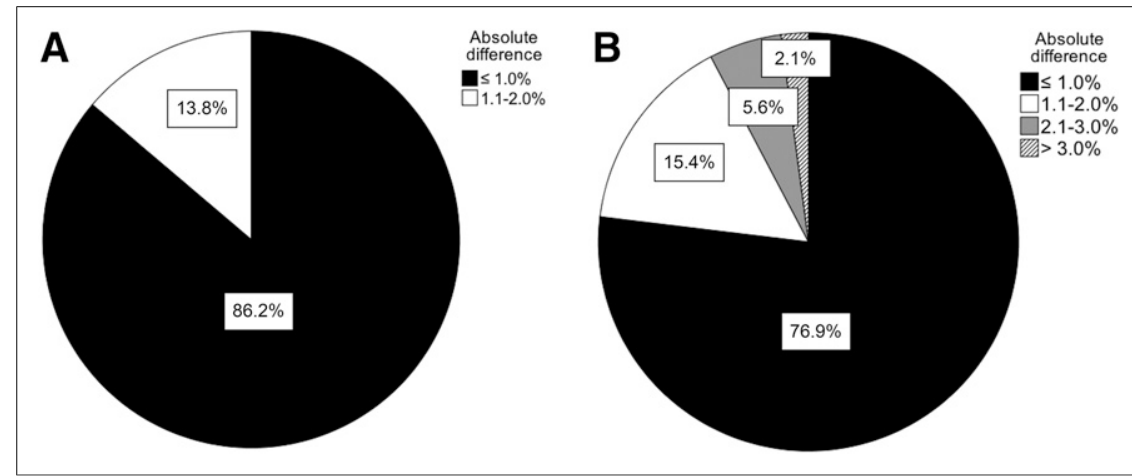


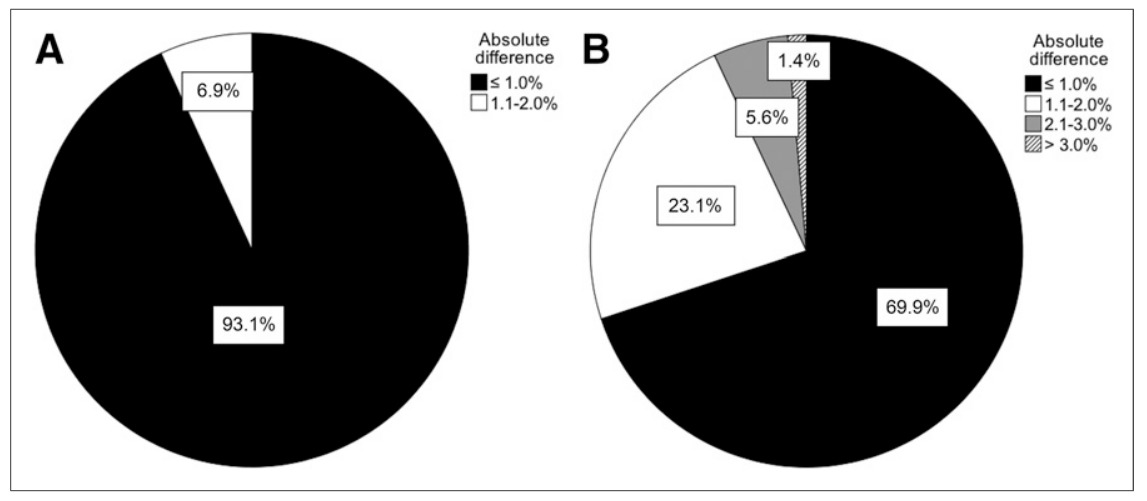

FIGURE 5. Absolute differences in intraobserver measurements of total lung $(A)$ and lobar $(B)$ contribution using SPECT/CT. it would have been possible to use a previous diagnostic CT scan and coregister it for lung segmentation; however, this was not done because we wanted to ensure uniformity of the method across patients and avoid differences in lung volumes between CT and SPECT acquisitions. Another limitation of the software is that the automatic lung contour detection is sometimes more difficult when lung density is highly heterogeneous, such as in the presence of extensive bullae mixed with areas of fibrosis. As previously mentioned, one patient had to be excluded because the software failed to detect the lung contours. This failure was found to be due to an endobronchial density that prevented the region-growing algorithm from functioning correctly. A disadvantage of SPECT/CT-based methods is dependence on tomographic acquisitions and hybrid cameras, which can increase imaging cost.

Limitations of this study also include a small number of patients, who were all nonsurgical candidates with a high prevalence of underlying lung disease. He et al. (9) have described the accurate prediction of postlobectomy lung function in 305 patients using a semiautomatic lung segmentation algorithm. Further work is needed to evaluate whether results obtained with the tridimensional technique predict the posttreatment forced expiratory volume in $1 \mathrm{~s}$ more accurately than traditional planar scintigraphy in patients undergoing radiotherapy. It would also be interesting to take into account the actual radiation treatment fields in quantification of regional parenchymal perfusion and prediction of posttherapy forced expiratory volume in $1 \mathrm{~s}$.

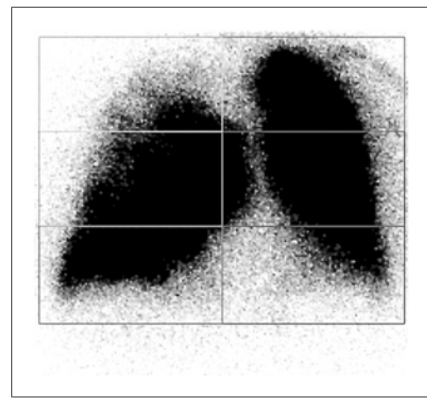

FIGURE 6. Example of inadequate segmentation on planar scintigraphy due to right lung parenchyma overlying midline. There is also prominent vascular activity, partly included in left upper and left middle lung regions (anterior view is shown; image contrast is increased).

\section{CONCLUSION}

Tridimensional lung lobar quantification can easily be performed using SPECT/CT acquisitions and semiautomatic segmentation software for both perfusion and ventilation studies. This segmentation technique demonstrates excellent interobserver and intraobserver agreement. Results obtained with this method differ significantly from those obtained with planar scintigraphy, especially for the right lung lobes. We recommend that SPECT/CT-based quantification be used for all lung cancer patients undergoing pretherapy evaluation of regional lung function. Further studies are needed to evaluate prediction of postradiotherapy forced expiratory volume in $1 \mathrm{~s}$ with this technique and whether quantification of perfusion or ventilation yields the most accurate results.

\section{DISCLOSURE}

The semiautomatic lung segmentation software (Hermes Hybrid 3D Lung Lobe Quantification) was provided by Hermes Medical Solutions with a prerelease agreement. No other potential conflict of interest relevant to this article was reported.

\section{REFERENCES}

1. Brunelli A, Kim AW, Berger KI, Addrizzo-Harris DJ. Physiologic evaluation of the patient with lung cancer being considered for resectional surgery: diagnosis and management of lung cancer, 3rd ed-American College of Chest Physicians evidence-based clinical practice guidelines. Chest. 2013;143(5 suppl):e166S-e190S.

2. Mazzone PJ. Preoperative evaluation of the lung cancer resection candidate. Expert Rev Respir Med. 2010;4:97-113.

3. Marks LB, Hollis D, Munley M, et al. The role of lung perfusion imaging in predicting the direction of radiation-induced changes in pulmonary function tests. Cancer. 2000;88:2135-2141.

4. Garrity ER. Clinical applications of pulmonary nuclear medicine. In: Henkin RE, Boya D, Dillehay GL, Karesh SM, Halama JR, Wagner RH, eds. Nuclear Medicine. 2nd ed. St. Louis, MO: Mosby; 2006:1349-1350.

5. Royal HD. Pulmonary imaging for nonthromboembolic disease In: Henkin RE, Boya D, Dillehay GL, Karesh SM, Halama JR, Wagner RH, eds. Nuclear Medicine. 2nd ed. St. Louis, MO: Mosby; 2006:1423-1438.

6. Knollmann D, Meyer A, Noack F, Schaefer W. CT-based 3D vs. planar derived quantification of pulmonary lobar perfusion in preoperative lung cancer patients with borderline lung function [abstract]. J Nucl Med. 2012;53(suppl 1):604

7. Kovaćevic-Kuśmierek K, Kozak J, Pryt L, et al. Perfusion lung scintigraphy for the prediction of postoperative residual pulmonary function in patients with lung cancer. Nucl Med Rev Cent East Eur. 2015;18:70-77. 
8. Toney LK, Wanner M, Miyaoka RS, Alessio AM, Wood DE, Vesselle H. Improved prediction of lobar perfusion contribution using technetium$99 \mathrm{~m}$-labeled macroaggregate of albumin single photon emission computed tomography/computed tomography with attenuation correction. $J$ Thorac Cardiovasc Surg. 2014;148:2345-2352.

9. He W, Zhai W, Avondo J. The clinical value of novel hybrid 3D lobar quantification SPECT lung ventilation/perfusion scan in predicting remaining lung function for lobectomy lung cancer patients [abstract]. J Nucl Med. 2016;57 (suppl 2):244.

10. Imaeda T, Kanematsu M, Asada S, et al. Prediction of pulmonary function after resection of primary lung cancer: utility of inhalation-perfusion SPECT imaging. Clin Nucl Med. 1995;20:792-799.

11. McGraw KO, Wong SP. Forming inferences about some intraclass correlation coefficients. Psychol Methods. 1996;1:30-46.

12. Bland JM, Altman DG. Statistical methods for assessing agreement between two methods of clinical measurement. Lancet. 1986;1:307-310.

13. Hirose Y, Imaeda T, Doi H, Kokubo M, Sakai S, Hirose H. Lung perfusion SPECT in predicting postoperative pulmonary function in lung cancer. Ann Nucl Med. 1993;7:123-126.

14. Knollmann D, Meyer A, Noack F, Schaefer WM. Preoperative assessment of relative pulmonary lobar perfusion fraction in lung cancer patients: a rather simple three-dimensional CT-based vs. planar image-derived quantification. $\mathrm{Nu}$ klearmedizin. 2015;54:178-182.

15. Piai DB, Quagliatto R Jr, Toro I, Cunha Neto C, Etchbehere E, Camargo E. The use of SPECT in preoperative assessment of patients with lung cancer. Eur Respir J. 2004;24:258-262.

16. Yin L, Shcherbinin S, Celler A, et al. Incorporating quantitative single photon emission computed tomography into radiation therapy treatment planning for lung cancer: impact of attenuation and scatter correction on the single photon emission computed tomography-weighted mean dose and functional lung segmentation. Int J Radiat Oncol Biol Phys. 2010;78: 587-594.

17. Yin Y, Chen JH, Li BS, et al. Protection of lung function by introducing single photon emission computed tomography lung perfusion image into radiotherapy plan of lung cancer. Chin Med J. 2009;122:509-513.

18. Knollmann D, Avondo J, Meyer A, Schaefer W. CT-based 3D quantification of relative pulmonary lobe perfusion from SPECT in preoperative lung cancer patients: comparing a manual and a semi-automatic approach [abstract]. $\mathrm{J} \mathrm{Nucl}$ Med. 2016;57(suppl 2):1731.

19. Thieme SF, Johnson TR, Reiser MF, Nikolaou K. Dual-energy lung perfusion computed tomography: a novel pulmonary functional imaging method. Semin Ultrasound CT MR. 2010;31:301-308. 